\title{
THE PERCEPTION AND THE RECOGNITION OF HUMAN FACES AND THEIR EMOTIONAL EXPRESSIONS - IN HEALTHY SUBJECTS AND SCHIZOPHRENIC PATIENTS
}

\author{
Vadim S. Rotenberg* \\ Tel-Aviv University, Bat-Yam, Israel
}

\begin{abstract}
This article displays a reconsideration of literary data that leads to the suggestion that the process of the face identification and of the recognition of human facial expressions, in addition to the perception of single facial features and their configurations, is based on the more essential creation of the polysemantic interpersonal relationships. In schizophrenic patients this ability is lost. The feeling of the human nature of faces is out of the competence of these patients and faces cause emotional tension with the subsequent use of the psychological defense.
\end{abstract}

Key words: Human facial expression; Interpersonal relationships; Psychological defense; Schizophrenia

\section{FACIAL IDENTIFICATION IN HEALTHY SUBJECTS}

This article is not a comprehensive review of the scientific publications related to the title. This article is a scientific essay that is going to discuss some questions and contradictions and to propose some speculative suggestions just with a hope that these speculations will be used for further investigations. I am going to discuss the psychological and brain mechanisms of face and facial expressions perception and recognition in healthy subjects and in schizophrenic patients. However, I hope to put this problem in a broad frame of human interrelationships.

In some investigations the process of face identification is considered to be a detection of some peculiar features of the face, like the shape and the size of nose, eyes, lips etc. (Joshua \& Rosell, 2009). This way of face identification, being based on the splitting of the face on its separate elements, is proposed as a natural. Probably it really may be relevant in those relatively rare cases when some particular facial features look out as so outstanding that can be used by itself for the recognition of the person. For instance, according to some contemporaries, the Russian famous producer Vsevolod Meyerchold had exactly such a nose: on one caricature his nose was looking out of the corner of the house and the inscription under the drawing was: Five minutes before the appearance of Meyerchold".

However, with exception of such exotic cases, human face is perceived as a holistic image, as a gestalt (Calder \& Jansen 2005). That's why, beyond stressing the importance of single features in face identification, scientists estimate the leading role of the general configuration of the face in this process, and they select figures of this configuration that are available for the

*Correspondence to: Vadim Rotenberg, email: vadir@post.tau.ac.il

Received October 28, 2010; accepted November 14, 2010; Act Nerv Super (Praha) 53(1-2), 1-20. 
algorhythmic analysis (Joshua \& Rosell, 2005). The basic configuration of the first order reflects the natural distribution of interrelationships between facial features: eyes are above the nose, nose is above the mouth. The configuration of the second order includes the space between particular facial features that are different in different persons: a distance between eyes, between nose and mouth, between eye brows and head hair, etc.

The articles on this topic make an impression that the recognition of faces is achieved on the basis of a very speed calculation and estimation of all these parameters, and only these configurations in combination with the single particular features are responsible for the distinguishing one face from another. Thus, the process of face identification can be computerized. However, it means that according to these parameters (items, figures) human face does not differ essentially from any object of the world (may be except of the basic configuration of the first order that is specific for the face). This basic configuration cannot be inverted because if eyes are under the nose it is not a face more. If the picture of the face is inverted and the forehead is localized downstairs and the chin - upstairs, healthy subjects usually have difficulties in face recognition, while the inversion of other objects, even very complicated according to their configuration, does not disturb the process of recognition (Yin, 1969). Thus, the basic configuration allows to discriminate any face from any object. However, it does not determine the recognition of any concrete face because according to these variables all faces are similar.

According to the configuration of the second order, that was suggested to be responsible for the recognition of the particular human face, human faces seem not to differ from objects. Probably it is another domain in the face that is closely related to the unique quality of personality, and is responsible for face recognition, but was not grasped by the investigators? Is a picture of the face drawn according to the formal rules (algorhythms) really similar to the picture drawn by an artist or at least similar to the photo of the vivid face? All these aspects of the problem are not discussed.

\section{THE RECOGNITION OF EMOTIONAL FACIAL EXPRESSIONS IN HEALTHY SUBJECTS}

Up to this point we have considered the recognition of faces with neutral expressions. However, people are recognizing not only different faces - the own face, faces of famous persons, familiar faces, unfamiliar faces of those persons they have met previously for occasion or those they are motivated to remember in the process of investigation. People are able to recognize also facial expressions of different emotions. Personal identity is invariant, while emotional expressions are different in the same subject. It was suggested that different brain systems are responsible for the recognition of the identity of the subject and for the estimation of his/her emotional expression (Haxby et al., 2000). In most of the investigations, scientists have checked the recognition of the very definite, doubtless emotional expressions, like oppositions between happiness and grief, happiness and fear, pleasure and anger etc. In some publications (Tsoi et al., 2008; Norton et al., 2009; Alimova et al., 2009) it was shown, that healthy people recognize positive emotional expressions (joy, pleasure, happiness) more easy than the expressions of surprise or of negative emotions (anger, fear, disgust, grief). The expression of happiness is recognized more easy, with a shorten delay, than the expression of surprise, expression of surprise - more easy than expression of anger, expression of anger more easy than expression of fear, fear more easy than grief, and grief is recognized more easy than disgust (Adolphs, 2002). However, some emotional expressions are often mixed with other - for instance, fear can be accepted as a surprise (Adolphs, 2002). The schedule for the recognition of happiness is lower than the schedule for the recognition of fear (Norton et al., 2009).

The difference in the recognition of different emotional expressions may be caused by two reasons. On the one hand, some emotional expressions, like happiness, may be more simple for recognition being based on some definite monosemantic features like lips that are stretched in a smile and are interpreted as a sign of positive emotions. 
However some recent investigations (Niedenthal et al., 2010) confirmed what all of us new from our own personal experience: a smile can reflect not only a state of joy and happiness, it may be a smile of superiority, a sign of politeness or an expression of irony. In the abovementioned investigations where positive facial expressions were compared with the negative one, smile was always used as an expression of happiness and joy - according to the conditions of these investigations. In the real communications healthy people are able to recognize all shades and meanings of smile, however it would be reasonable to check whether the smile of superiority is recognized as easy as smile of happiness and whether the recognition of superiority (that displays itself in smile) is also more easy than the recognition of fear and anger. And if the significant difference is only between the recognition of the true happiness and negative emotions, it would mean that it is not the formal shape of lips (smile) that determines the prevalent recognition of positive emotions. It would mean that it is another mechanism. It can be a psychological defense mechanism (denial or repression) that protect subject from the perception of negative face expression but has no reason to protect subject from positive face expressions. Some authors (Norton et al., 2009; Huang et al., 2009) suggest this mechanism to explain the more high schedule for recognizing negative emotional expressions in schizophrenia (see later) but the similar mechanism may act in healthy sensitive subjects. Anger of another person may cause fear; melancholy or disgust may be passed to the person who percepts these expressions and a person may try to protect himself from such emotions. Quite opposite, a person would be glad to share with another person a feeling of joy and it makes the schedule for this expression more low.

Bar et al. (2006a) have shown that people prefer soft and circular visual objects vs. sharp and full of corners that arose a feeling of danger. The same is with the face images - their circular and soft shape provokes sympathy and tenderness and became a trigger of positive emotions. Niedenthal et al. (2010) suggest that those who are viewing a smile of happiness are creating in their mind an "embodied reproduction" of the positive affective state transferred by the smile, and due to this reproduction of affect understand the meaning of a smile of smiling person. This "embodied reproduction" from my point of view reflects the emotional contact based on polysemantic relationships with the smiling person. Niedenthal et al. suggest (and this suggestion is very reasonable) that the analysis of the features of the face may be sufficient for the estimation of the conventional and standard face expressions in simple tasks (when smiling lips reflect joy and frown brow reflect anger). However, such formal analysis does not help to recognize vivid expressions that contain all shades of feelings, as well as for the representation their fine and ambiguous nuances. For such tasks it is not enough to get a direct visual perception of the particular features of the face and their configuration. It is necessary to build a more broad context that includes these features of the face but has something in addition. For instance, this context has to include some knowledge about a person who express emotions, or to guess about it on the basis of previous experience, for the relevant interpretation of the ambiguous face expression. This expression became "embodied" in the motor, somatosensoric and emotional systems of the observer. Affect that is caused by another person, is projected on this person.

Human face is different from all other objects because it includes the imprinting of the unrestricted rich personality of the subject that is in complicated and polydimensional relationships with the polydimensional and polysemantic world (Rotenberg, 2007). Due to these polysemantic relationships the holistic perception of the face, as well as the perception of the vivid personality as being an owner of this face, cannot be reduced to the perception of the particular features of this face, their combinations and configurations. Such a holistic perception means to overcome the restrictions of these features and configurations and to include an unlimited spectrum of relations between subject and the world. Just due to the perception of the face in this broad context, the observer is able to conclude, while looking on the neutral face expression without definite signs of anger, fear, disgust or joy, whether the owner of this face is dangerous or not, is he/she pleasant or not, - and it is difficult for observer to explain the reasons of this implicit and integrative conclusion. 
Bar et al. (2006b) have shown that the first and most important impressions evoked by personality appear very soon, in 39 msec., on the early stage of the face perception. During this very short period the estimation of the threat caused by the face with emotionally neutral expression was related to the personality of this unfamiliar person per se, not to his/her temporary emotional state. This personality is estimated, for instance, as dangerous in general, does not matter whether it was threatening for the observer in this particular moment, because the estimation was performed not in the process of the direct contact with this person but only by viewing his/her grey - even not coloured !- photography with a neutral expression. Authors emphasized that the features of the face that caused threatening vs. non-threatening impression are grasped very quickly because this impression has a vital meaning (sense) and is important for the security of the observer. I suggest that such impression is determined not by particular features of the face (at least in most cases when they are not particularly distorted) but by the broad spectrum of past associations based on the previous experience. Ghuman and Bar (2006) have emphasized in their investigation the role of these associations in the estimation of visual objects, and it seems to be especially important for the estimation of the face. It reflects the integration of the human face into the net of polydimensional relationships. If, for instance, we are coming back to the problem of the perception of the smile, there are no formal criterions for its truthful sense - whether it is accepted as a truthful or false is determined by the contextual relations and associations. Due to these relationships a smile, a face impression and a body posture contain something more than what can be analyzed according to the formal criterions. Probably as I have mentioned, for the formation of the relevant contextually determined associations is necessary to rely on the previous experience of interpersonal relationships. But presumably there may be also some inherent predispositions to this ability: an infant is searching for the contact with the empathic person and is crying while watching even a neutral expression of the face of the emotionally blunt person. It means that even an infant is able to grasp the subject's ability to build emotional relationships due to the functional domination of the right hemisphere, its temporal lobe (Saugstad, 1998). With the warm mother such relationships start almost immediately after birth. However, in the process of maturation this ability may develop or decrease probably according to the domination of the right-hemispheric or left-hemispheric mentality (what also partly depends on the character of relationships with the surrounding social environment). It is important to realize that the smile (as well as any other facial expression) is not simply "embodied" in the observer, but between two persons appear new polysemantic interrelationships.

The smile of the own and of the strange (somebody else's) child both are usually causing positive emotional reaction, however they cause different reactions in the fronto-orbital cortex of the adult (see Niedenthal et al., 2010). It confirms the conclusion that it is not a simple reaction on the smile per se, but on the smile that is integrated in the relationships with the subject's own child. The same happens between adults. A subject perceives and estimates a face of another subject after he has seen during his life numerous different faces and they have made different impressions. Emotional tension that the sensible person often suffers from in the crowd may be also a reaction on this stream of associations. With some persons have been previously created emotional relationships, and the facial expressions of some strangers may resemble such emotional relationships even vague.

Mimicry is a visible or un-visible use of subject's own mimic muscles of observer in order to compare it implicitly with a facial gesture of observed person (see Niedenthal et al., 2010). It is possible to suggest that implicit imitation of the someone's facial expression helps subject to understand the sense of this expression not only because the reproduction of the mimic reaction activates brain zones that are responsible for the relevant experience. When subject is reacting on the face expression of another subject he/she is not only activating his/her limbic system through the own facial muscles - he/she is creating (unconsciously) new relationships with another person. Would the outcome be the same if subject will try to imitate the someone's facial expression using his own mimic muscles without the direct visual contact, only according to verbal instruction?...It seems very doubtful. 
The mimicry of the facial expression may be important because it is a sign for the observer that he/she started an emotional contact. When the facial mimicry is blocked, emotions became less prominent.

In this context it is interesting that the organic paralysis of the mimic muscles of observer do not prevent the recognition of emotional expression of the observed person, while the pencil in the mouth that also blocks the mimic imitation disturbs the recognition of emotional expression (see Niedenthal et al., 2010). It means that the problem is not the blockade of facial muscles per se; the artificial situation caused by the pencil in the mouth shifts the attention of the observer and makes his/her interrelationships with the observed person unnatural while the stable paralysis of facial muscles causes finally the psychological adaptation to this condition. Thus the mimicry of facial expression participates in the interpersonal relationships.

The active participation in emotional polysemantic relationships is from my point of view more important for the recognition of emotions than the feedback between mimic muscles and brain zones of emotional reactions. I suppose that if person will simply learn some mimic reactions it will not cause emotional experience that is relevant to these reactions in the natural conditions.

At the same time, in the process of recognition of the typical standard facial expressions mimicry may be less important because such recognition includes conceptual knowledge instead of the vivid interrelationships. However, mimicry may be not very important also when the polysemantic interrelationships between observer and observed subject are available without it.

When the observer gets an instruction to lower voluntary the corners of the mouth it does not block the recognition of the observed facial expression. This result of the experimental investigations (see Niedenthal et al., 2010) needs explanation. One such speculative explanation is that to lower the corner of the mouth is a formal and easy voluntary gesture that does not allow to imitate smile but it does not block the concentration on this smile and the feeling of empathy and of course does not prevent the conceptual approach to the smile.

At the same time, a spontaneous imitation of the facial expression is a sign of empathy that is an important part of interpersonal relationships (see Niedenthal et al., 2010). Is empathy obligate for feeling another person? Autistic subjects who are not imitating facial expressions of other people but in general are functioning well are able to recognize facial expression, as well as healthy subjects do (see Niedenthal et al., 2010). These data require a careful analysis. Probably in experiments with autistic subjects the task (facial expression) was simple and standard? If it was the case, autistic subjects were oriented on formal concepts of facial expressions, not on the creation of emotional relationships, because in the every-day life they are not very sensitive to emotional variables.

The posture and gestures that are not directly expressed in mimic are also important for the recognition of the smile. They are also based on the right hemispheric mechanisms responsible for the polysemantic interpersonal relationships. Especially important is the eyes contact. Persons are more able to imitate the expression of pain when they are in a state of eyes contact and angry faces cause more negative affect in observer when the eye contact is present. The expression of human eyes is inexhaustible. However, those who percept the smile of pleasure can smile back without eye contact with the observed smiling person, simply due to positive emotion caused by the smile per se. Such a reaction - smile towards the smile of somebody - is the reaction on the joy of another person, even without the direct contact with this person. When the smile reflects only the joy initiated by the joy of another person, this smile does not differ from the smile on any positive information and does not reflect the interpersonal contact. Such smile also may not be accompanied by the eye contact. However, in general eye contact is an important sign of humanness that includes the polysemantic relationships between people.

A three-day old child is viewing faces with a gaze that is directed on him for a more long period than faces with a gaze turned away from him (see Niedenthal et al., 2010). However, is it the same eye contact as in adults?...An infant is grasping an integrated holistic face 
expression in order to understand (on the unconscious level) whether it is dangerous or not, comfortable or not to be in contact with this person. It is a monosemantic impression, and the reaction of the infant on this holistic message is also monosemantic. For the polysemantic emotional contact the infant still has no skills. Autistic children avoid direct eyes contact in the process of recognition of emotional facial expression however they are often able to recognize these expressions correctly probably due to orientation on other parts of the face (see Niedenthal et al., 2010). A normal adult subject may avoid eyes contact if he/she is afraid of emotional interrelationships and do not feel himself confident in such relationships. However, this subject is able to recognize correctly a relatively simple face expression. Inability to have a direct eyes contact may increase attention to the lips region and due to this attention subject is able to discriminate the expression of joy from the expression of fear or disgust.

Eyes contact is working as a trigger for the embodied simulation that prescribes to the smile different and vague functional meanings (see Niedenthal et al., 2010). Exactly the eyes contact is leading to the embodied simulation because eyes contain a rich world of experiences that do not have formal expression. Eyes contact and embodied simulation (imitation) may become repressed if they seems to be too risky or even aversive. When the eyes contact is absent or when embodied simulation that reflects the polysemantic contact between people is suppressed by social factors, the estimation of the sense of the smile (whether it is true or false) is determined by monosemantic stereotypes that have social roots. People often use such stereotypes and concepts that are creating a probability forecast according to the meaning of the smile. Due to such concepts people are able to translate an experience elicited by the direct perception into the amodal language. (Niedental et al., 2010). Polymodal perception is not equal to its conceptual presentation. Different systems are responsible for them.

According to my theory (Rotenberg, 1979, 1985, 1994, 2004, 2007, 2008) the difference between systems is related to the difference between the right hemispheric way of thinking that creates the polysemantic context and left hemispheric way of thinking that is responsible for the formation of the monosemantic context. Of course the concept may influence the perception by activating a previous experience however a new direct impression may be more strong...

From my point of view, emotions may be created by the concepts, however if they are not supported by the direct actual experience or at least by the previous experience of vivid feelings they are not complete and sufficient. Of course initial concept may influence the direct perception ( the perception of the smile) but this concept has to be based on the relevant past experience that forms the vivid image what the smile has to express and how it has to look out. For the real embodied simulation it is necessary to overcome restrictions of the monosemantic context (if this context is not triggering the vivid past experience, but in this condition it is not a pure concept). Does it not mean that the pure concept may become a trigger that activates mechanisms that are abolishing this concept?...

In one investigation (see Niedenthal et al., 2010) subjects have watched natural real smiles and they were informed that these smiles appear either in the context that makes natural smiles predictable, according to the cultural stereotypes, or that these smiles appear in the context that predispose to the false smiles. In half of investigated subjects the facial imitation of the watched smiles was artificially blocked. All subjects had to estimate the degree of the sincerity of the watched smile.

When the imitation of the smile was available, the information of the cultural stereotypes was not influencing the perception of the sincerity of the smile probably because the imitation helped to create interpersonal relationships and empathy that overcome the restriction of the concept. When the imitation was not available, a smile that appeared in the context associated with a natural smile was accepted as a more natural than smile that appeared in the context that made false smiles principally available. However, actually all smiles were natural but when the imitation is blocked a context and a concept are influencing the perception. It means that imitation makes the direct emotional interrelationships more 
available and more effective. (When I speak about emotional interrelationships it does not mean of course that the observed subject especially being on the picture is really participating in these relationships. However it does not prevent the feeling of the observer that he is in emotional relationship with the observed subject, like it happened when we see on the street a happy unfamiliar subject and feel that we share for a moment a happiness with him or when we feel close relationships with a person on the picture of a great artist.) The embodied simulation that Niedenthal et al. are speaking about is an empathic resonance and it is critical for the estimation of facial expression. The reaction on the smile with subject's own smile is a personal participation in collaboration. It is something more broad and essential than the estimation of the emotional state of another person. It makes a person no strange, no more alien. It is related to the personality of the observer. And the identification of faces - not only of the facial expressions - presumably includes the same mechanism because every face if it is vivid produces an aura of polysemantic contextual associations.

In order to be perceived, emotional expression of the face has to make a holistic impression on the observer. A composition from the joyful and angry expression of the same face ( when eyes express an anger and lips express a joy) makes the recognition of such combined expression difficult and takes more time even if observer is required to concentrate attention consequently on every half of this combined photo. It is difficult for the observer to ignore the impression caused by the holistic gestalt of the face, as well as it is difficult to recognize a person on the photo combined from the parts of faces of two different persons with neutral faces expressions. (Calder et al., 2000; Young et al, 1987). At the same time, this disturbing effect of composition is absent, if the photo is combined from the pictures of two different persons with the similar emotional expressions and observer has to recognize only the emotional expression (Calder et al., 2000). In such case the combination of the upper part of the face picture of one person with the lower part of the face picture of another person does not disturb the recognition of the facial expression. Moreover, even the face by itself is in this case estimated as belonging to one person. It means that emotional expression is a determinative factor probably because the emotional expression is relatively more simple and definite than something that determines the recognition of personality, especially if this person is unfamiliar for the observer and is not famous (it means not well-known in the society). Another reason of such domination of emotional expression may be the importance of the emotional expression in human's interrelationships. However, it is necessary to take into consideration that emotional expressions used in such investigations are in most cases based on a very definite and standard, almost monosemantic mimic expressions (like broad smile of lips- smile of joy).

The mechanisms of the recognition of simple emotional expressions that can be conceptualized and mechanisms of face recognition are different. (Calder \& Young, 2005; Haxby et al., 2000; Schwartz, 2002; Pomarol-Clotet et al., 2009). That's why during the recognition of emotions the difference between two parts of the face picture that belong to different persons is ignored if emotional expressions of these two parts are similar, and these two parts are not estimated as belonging to different subjects. At the same time, when it is necessary to recognize a person and two different emotional expressions of the same person are combined, the recognition is available, because the signs of personality in this task are more important. Actually, we are able to recognize the same subject with different emotional expressions probably until the emotional experiences caused by his life are not changing the personality by itself. The role of the complicated but unified image is probably less important in the recognition of emotions (especially of relatively simple emotional expressions) than in the recognition of personality.

However, both mechanisms are probably not totally separated (see Calder \& Young, 2005). The recognition of the complicated emotions requires right-hemispheric mechanisms that are responsible for accepting the polysemantic context and are also used in the process of face recognition. On the other hand, during a face recognition, as I have mentioned, appears a thin connection between the past experience of observer, including his/her emotional associations, and the image of the person who is an object of observation. Actually it is 
something more than the recognition - it is an inclusion of the image of observed person into the broad mental world of the observer. Human face contains something for another subject that makes this face very special, and it is impossible to reduce this particular meaning to the list of separate features and even to their configurations. Face is perceived as a meaningful gestalt and authors emphasize (Calder \& Jansen, 2005 ) that the holistic recognition of the face as well as of the natural, not conceptualized, emotional expression are not explained by the configurations of the second type (see above). Face is not an object.

The reaction of the brain on the holistic image of the face (global processing) forestalls its reaction on details of this image (Obayashi et al., 2009). The integrative perception of the image is performed by the right hemisphere more speed than the analysis of its parts (Kostandov \& Genkina, 1976). However, the mystery of this holistic perception of the face is that it is determined by something what is beyond this image per se, by something that integrates it in the broad context of the inner world of observer. I have already emphasized that even a fluent view on the face of the unfamiliar subject with a neutral face expression may elicit in observer a feeling of empathy or an opposite feeling, and it is an outcome of the holistic impression that creates the image of the face not as an image of a separate object but as a vivid image that is already in some mysterious relationships with the observer.

In this context it is interesting to mention that in dogs experts who are training dogs the effect of inversion of the muzzle of the dog is similar to the effect of the inversion of the human face (see Schwartz et al., 2002). It does not happened in non-experts in dogs. It is possible to speculate that these specialists create personal relationships with their dogs that are absent in other persons.

\section{THE ROLE OF THE SOCIAL STEREOTYPES}

Simons and Levin (1998) have shown that the perception and recognition of another subject depends on whether the observer consider the observed subject to belong to his own social group. In their investigation an experimenter initiated a conversation with a pedestrian and during the interaction he (investigator) was surreptitiously replaced by another experimenter. When the experimenters appeared to be members of an out-group the ability to detect a change to the centrally attended object in a scene was dramatically reduced. The experimenters imitated construction workers, and a pedestrian (a student) categorized them as construction workers. By this formal categorization subject did not retain those personal features that would allow individuation. No personal relationships were created with this occasional "construction worker". Subjects appeared focused on the interaction and conversation, often making even eye contact with the experimenters, listening to their voices, and all these interrelations did not help subject to discover the replace of the experimenter by another one, because the conversation does not include the personal contact - subject contacted with a concept of "construction worker'. The observer (pedestrian) was not considering the experimenter to belong to his own virtual world. It means that when subject considers another person with whom he is communicating not to belong to his representative group (according to the age, social position, nation etc.) he often perceives this person differently, not like an individual person but like a representative of a concept, and may be not able to recognize him."

\section{THE RECOGNITION OF THE OWN FACE}

A very important part of the problem is the recognition of the subject's own face and its difference from the recognition of familiar faces of other persons. According to Keenan et al. (1999) if a person sends a signal with his right hand that he recognized on the photo himself vs. another but familiar person, these two reactions do not differ according to the delay. It 
means that for the left hemisphere being responsible for the decision in this condition the subject's own face and the face of the familiar person are equal.

However, when the same subjects reacted with their left hands (and the right hemisphere was responsible for the decision and action) the identification of the familiar faces was performed with a greater delay in comparison to the own face identification. Authors conclude that self-recognition is performed by the right hemisphere. From my point of view it does not mean that the right hemisphere is not participating in the recognition of familiar faces, but probably for this task the role of the left hemisphere is also important. At the same time Self-Image localized in the right hemisphere (Rotenberg, 1982) is presumably responsible for the recognition of the subject's own face. Self-Image integrates numerous connections between the person and the world that includes objects, events, social interrelationships, virtual world of culture, and due to all these connections and interrelationships integrates also the past and the present of the subject. And the left hemisphere is responsible for the Self-Concept that principally does not differ from any other concept, including concepts of the familiar and famous persons. The role of the Self-Image and Self-Concept in the process of self-recognition is different but both are participating in this process. Platek et al. (2008) have shown that although the right hemisphere dominates in the process of the self-face perception, some definite structures of the left hemisphere are also activated, particularly left fusiform gyrus.

Turk et al. (2002) investigated the patient with a split brain after cutting corpus callosum. Authors presented to his right and left hemisphere consequently his own face and faces of familiar persons. All these presented faces have been partly artificially distorted and the degree of such distortion was different: some pictures were very similar to the initial natural picture (image) but other were quite different. The percentage of the correct recognitions was of course higher when pictures were relatively more similar to the not distorted model, does not matter whether it was subject's own face or familiar face and the left or the right hemisphere was involved. Authors come to the conclusion that both hemispheres are able to recognize own and familiar faces. Moreover, recognition of the partly distorted own face was even more successful if it was presented to the left hemisphere. It seems to be in contradiction with the abovementioned data and data of other authors (Sugiura et al., 2005; Platek et al., 2004) that have shown the advantage of the right hemisphere in the process of own face recognition. However, it is possible to suggest that distortion of the face image disturbed the holistic nature of the image and this nature is obligate for the successful face recognition by the right hemisphere. As a result the advantage is on the side of the left hemisphere that is performing a consequential detailed analysis of the picture and compares the outcome of this analysis with the initial monosemantic concept. The attention to particular features, the ability to distinguish some details from the whole picture and to ignore them may explain the role of the left hemisphere in this task. Platek et al. (2004) belief that in this condition the task of the own face recognition as a holistic image is displaced by the task on the working memory. As soon as the opportunity to grasp the holistic image appears, the advantage turns back to the right hemisphere.

When in the presented face image the features of the own face have been artificially mixed with features of the face of well-known famous figures (Keenan et al., 2001) and the right hemisphere was suppressed for a short period, the observer took this image as a face of a famous person. When the left hemisphere was suppressed the observer recognized this mixed image as his own face - the general impression was more important than the single features. Presumably it is an additional sign of the role of the right-hemispheric Self-Image in self recognition. The middle frontal gyri of the right hemisphere plays an important role in the perception of the subject's own face and at the same time - in the empathic understanding of other subjects and in the perception of himself in the context of relationships with other people (Platek et al., 2008). When the image of the subject's own face is presented to the right hemisphere of the patient with a split brain, his galvanic skin resistance (GSR) became more prominent in comparison to the reaction on images of other faces or to the reaction on image of the own face presented to the left hemisphere (Preilowski, 
1977). Even words that characterize subject are activating the right frontal lobe (Craik et al., 1999).

The identification of the face and the recognition of its emotional expression have some common roots: it is a polysemantic context of relationships not only between particular features of the face, but also between this face and the world, including the inner world of the observed subject and of observer. It makes a difference between the identification of the human face and the identification of the inanimate objects - the former is an identification of the dynamic essence, not a harden essence. Just due to this dynamic vivid essence a person can become not recognizable although the configuration of his/her face does not change. And at the same time the person can be recognizable nevertheless of very different emotional expressions on the face if person is the same according to his essence. Thus when subject suddenly became unrecognizable his/her relatives intuitively feel that he/she changed as a person, it means the all his/her relationships with the world and other persons changed.

This theoretical approach is supported also by the data of the investigations that have shown that prescribing to another subject a definite psychic state and the understanding of the mental state of another subject is determined by the activity of the same zones of the right hemisphere (the middle and the upper frontal gyrus) that are responsible for the recognition of the own face (Platek et al., 2004). According to Gallap (1982), in order to recognize the own face in the mirror it is necessary to build a Self-Concept .

The formation of the Self-Concept and Self-Image has the following dynamic. In the early childhood a man has a simple holistic image of the world and of himself, and it corresponds to the initial domination of the right hemisphere. This simple sensation of himself as a being that may have pleasant and unpleasant feelings have an infant and a monkey. However it is neither Self-Concept nor Self-Image. Self-Concept appears in parallel with the development of the logical way of thinking, and in parallel develops the self-separation from the world and even the opposition to the world, and relationships with the world became restricted and impoverished. Consequently appears a need in the secondary integration in the world, already in a new position of being an independent subject. It is a need in creation of the new polysemantic relationships with the world that overcome this position of separation and opposition to the world. This task is achieved due to the development of the frontal lobe of the right hemisphere that is the latest brain zone to be matured and is the basic structure of the highest mental functions (Rotenberg, 2004, 2007).

The damage of the right hemisphere, more often than the damage of the left hemisphere, causes the inability to interpret the mental state of another person and to understand jokes that contain heroes (Happe et al., 1999). It means that the virtual relationships with heroes are disturbed. It is difficult to such patients to imagine the ideas of others that are opposite to the well known "final truth". The empathy and the ability to understand the psychic state of another person (Theory of Mind) are related to the feeling of being integrated in the polydimensional complicated world where another person is integrated in also - a logical concept for such feeling is not enough. The damage of the left hemisphere does not disturb this empathy feeling of another person (Happe et al., 1999).

Of course, the understanding of another person may be based on the logical analysis also, and these two ways of understanding can complete each other. However, inability to imagine the perception of the other person on the basis of subject's own experience (Stuss et al., 2001) is first of all the inability to identify himself with this person and not a deficiency of analytical skills. Although the right hemisphere is responsible for both the perception of the visualspatial information and for the integration in the world, these two functions are different and are performed by different structures of the right hemisphere. For instance, in autism the visual spatial perception is not disturbed while skills of the global perception of the gestalt are absent. 


\section{FACE AND FACE EXPRESSION IDENTIFICATION IN SCHIZOPHRENIC PATIENTS}

Schizophrenic patients have problems with face identification and make more mistakes in this domain than healthy subjects. In one investigation (Shin et al., 2008) subjects had to discriminate faces presented on photo. Faces have been presented in normal and inverted positions. Subject had to estimate whether two faces presented in parallel ( at the same time ) are identical. Authors come to the conclusion that in comparison to healthy subjects patients are less able to recognize the configuration of faces while particular facial features were perceived normally.

However, not all investigators agree with this conclusion. Schwartz et al. (2002), have shown that in healthy subjects and in schizophrenic patients the alteration of the configuration of the face is similarly influencing the process of face recognition, and suggested that schizophrenic patients are also using the configuration of the face for its recognition.

In healthy subjects the inversion of the face changes the perception of the basic configuration of the face as a holistic gestalt but not the perception of single features. In schizophrenic patients face inversion paradoxically disturbs face perception much less probably because in patients the perception of the face configuration is disturbed even in the face normal position, before inversion. In healthy subjects but not in schizophrenic patients the inversion of the face disturbs the perception more than the inversion of the object. Inverted faces are recognized on the basis of their particular features, and attention to these features restricts the perception of the face as a holistic gestalt. The inversion disturbs the integrated vivid impression made by the face because the face cannot be inverted - with the inverted face the observer is unable to create direct personal relationships, the inverted face is not accepted as a face of a subject. And if patients perceive faces similarly in a normal and in the inverted position probably it means that a "human" quality of the face is senseless for them.

It may be also the reason why schizophrenic patients perceive irregular transfigured faces as less strange in comparison to healthy subjects (Bleich-Cohen et al., 2009). If patients are not sensitive enough to such peculiarities of the normal, regular images of faces as its vividness, than the lack of the sensitivity to its transfiguration is not surprising. It is interesting that the fusiform gyrus reacts to the inverted faces similarly in patients and in healthy subjects (for whom the inverted face looses its quality as a face), and patients react in a similar way also on the non-inverted faces. Only in patients fusiform gyrus displays the same decrease of its physiological activity after the repetitive presentation of the irregular face and after the repetitive presentation of the regular face. It means that for the patients it is not very important whether the face is irregular or not, and they are more ready to perceive the irregular transfigured face as a normal one because they are unable to discriminate a normal face from an irregular. In patients in comparison to healthy subjects fusiform gyrus is less functionally connected with amygdala and prefrontal cortex and it may be related to the absence of the normal emotional reaction on the face. In healthy subjects the effect of the repetition of the stimulus (habituation) is decreased when the face is irregular and is estimated as a strange or unpleasant.

In healthy subjects the oxygenation of the right fusiform face area is more prominent in the process of the retardation (remembering) of faces that are afterward more easy recognized, while in schizophrenic patients this difference is absent (Walther et al., 2009). While recognizing famous and unfamiliar faces schizophrenic patients display less oxygenation in these structures, the performance is worse, the reaction time is longer. The evoked potential N170 that in healthy subjects appear on the early stages of the identification of human faces, in schizophrenic patients is similar in the process of face and object identification (Obayashi et al., 2009). It means that the face is perceived as any other object. In healthy subjects when they have to remember a face the FMRI amplitude is higher than when they have to remember chairs. In schizophrenic patients this difference is absent (Walther et al., 2009). In healthy subjects the amplitude is higher during the correct recognition in 
comparison to false recognition. In patients such difference is absent. In the process of the recognition of famous faces in patients the reaction time is increased, as in the false recognition of the unfamiliar faces. Schizophrenic patients are characterized by the decreased sensitivity to the low-rate stimulation that is especially important on the early stage of face recognition (Obayashi et al., 2009).

It is necessary to take into consideration that schizophrenic patients have problems in integration of the fragments into the holistic image not only during face perception but also during perception of the more simple objects (Doniger et al., 2001, 2002). They need more information for the correct identification of objects, and they are more sensitive to the visual backward masking of objects. When patients have to reproduce a straight line that contains "occasional" gaps they, in contrast to healthy subjects, do not fill in these gaps automatically and are reproducing them more correct - their imagination does not fill up these gaps (Snyder et al., 1961).

Despite of the all differences from healthy subjects, the performance of the task in the schizophrenic patients is improved as in healthy subjects when before performing face or object recognition they get pictures or verbal information that are relevant to the task (Doniger et al., 2001). It is possible to suggest that the presentation of these additional stimulus helps to create a concept that promotes the perception. However, according to some investigations (Tremea et al., 2009) schizophrenic patients often prescribe to these predictive signals an irrelevant meaning: signals that objectively are not dangerous are accepted as dangerous, while signals that are really signs of danger are not accepted as such. Schizophrenic patients often prescribe negative emotions like fear or disgust to the neutral face expressions (Kohler et al., 2003).

A perception closure can explain the inability to build the holistic object by using the perceived information and imagination combined with previous experience. Promotions that are helping to form a concept of the whole (I emphasize - concept instead of gestalt) may partly replace this non-integrated previous experience. Probably the construction of the conditional monosemantic concept based on the recognition of single features that are often repeated in the process of perception help to recognize faces of famous persons. The often repetition of the same visual expressions may explain also the successful recognition of the subject's own face (Pomarol-Clotet et al., 2009). However, schizophrenic patients spend more time for the recognition of their own faces than for the identification of familiar faces (Platek et al., 2002). It may be explained by the deficiency of the Self-Image. Schizophrenic patients often react on their own image in the mirror as if it is somebody else, somebody who exists independently (Harrington et al., 1989). They have also problems in recognition their own body image (Daprati et al., 1997). At the same time the inanimate objects are perceived normally (Orbach et al., 1966). Close relatives of schizophrenic patients also need more time than healthy subjects to recognize the self face, however in general they are more able to differentiate themselves from others. And patients periodically perceive their image in the mirror as a face of somebody else, even of somebody unknown (Platek et al., 2008).

The difference between healthy subjects and persons with a high level of schizothypy display itself also in some motor reactions. When healthy people are reacting on the adjectives that relate to them their left hand reacts more speed than the right one (Platek et al., 2003) but in subjects with a high level of schizopthypy this advantage of the left hand is absent.

In the investigation of Irani et al. (2006) schizophrenic patients, their relatives and healthy subjects have been asked to estimate as speed as possible whether the presented picture is their own face, the face of the unknown (unfamiliar) subject or a face of the familiar subject. The recognition of the own face was most successful in a healthy subjects and less successful in patients.

It was also found that only those subjects who are able to recognize themselves in the mirror are able to prescribe the mental state to another person in a relevant way. Although patients who do not recognize their own face are able to recognize the familiar faces, it is difficult for them to recognize unfamiliar faces if they have seen them only once before - 
more difficult than for healthy subjects. When they were unable to recognize their own faces but recognized the familiar faces they often were surprised while looking in the mirror: "Is it really I? I supposed that it is somebody whom I know." (Platek et al., 2004). Thus, it confirms the disturbance of Self-Image combined with the preservation of autobiographical memory.

Face inversion disturbed the perception of the face expression in patients less than in healthy subjects (Bleich-Cohen et al., 2009). Schizophrenic patients perceived the distorted face as less strange, in comparison to healthy subjects. As I have already proposed, it may be a sign that patients even without inversion perceive a face as an object without penetrating in its human nature. In such condition it is also impossible to build special human empathic interpersonal relationships that help to experience emotions of other person as subject's own emotions. Consequently, emotions can be recognized only by using the initial concept that connects definite configurations and features of the face with definite emotions.

The investigations of the identification of facial emotional expressions in schizophrenic patients contain many contradictions. In some investigations was shown that schizophrenic patients are more sensitive to positive emotional expressions vs. negative (Kohler et al., 2003; Bleich-Cohen, et al., 2009; Silver et al., 2009) . Accuracy in identifying sad faces was lower than of happy faces (Silver et al., 2009). Schizophrenic patients exhibited lower scores for open-mouth angry expressions (Leppanen et al., 2008). They displayed cognitive avoidance of negative stimuli (Morris et al., 2006). Definite fear antecedents were more frequently judged by schizophrenic patients as non-emotional, in comparison to healthy subjects (Tremea et al., 2009). Walker et al. (1980) have found in schizophrenic patients greater deficit in recognition of negative emotions. In opposite to healthy subjects who display a highest amplitude of evoked potentials while observing the negative emotional face expressions (Caharel et al., 2005; An et al., 2003) schizophrenic patients are more sensitive to the expression of happiness than to the expression of sadness. However this difference disappears when negative symptoms dominate in the clinical picture, and in any case patients are recognizing relatively simple emotions like sadness and joy worse than healthy subjects do (Turetsky et al., 2007).

Other investigators have found that patients in general more often estimate a face as a sad one (Tsoi et al., 2008). According to Turetsky et al., 2007, the amplitude of the N170 response to sad faces was in patients inversely associated with the severity of positive symptoms and with presence of delusions, and it corresponds to the above mentioned data of the same authors that the more prominent reaction on the expression of happiness vs. sadness disappears with the domination of negative symptoms. Thus, when Turetsky et al. (2007) conclude that happy faces where easier to identify for schizophrenic patients it means that their patients were characterized by the more prominent positive symptoms.

However, there are also opposite findings (Seiferth et al., 2009) that schizophrenic patients are characterized by the increased vulnerability to negative emotions, and delusions increase sensitivity to fear. It was also shown that the recognition of sadness is less impaired in schizophrenic patients (35) as well as that they display the decreased ability to recognize happy faces in contrast to sad and fearful faces (Tsoi et al., 2008). Tsoi et al. (2008) discovered in patients tendency to prescribe fear and sadness to any facial expression and to perceive neutral expressions as unpleasant, together with the decreased sensitivity to happy faces. According to (Seiferth et al., 2009) young schizophrenic patients reacted faster than they healthy coevals when identifying sad faces, but they were impaired in specificity when discriminating these faces. It means that even faces that were not definitely sad have been identified as such. In concordance with this investigation are data (Tremea et al., 2009) that by schizophrenic patients non-fear antecedents are more frequently judged as fear antecedents, and data of Kohler et al. (2003) that schizophrenic patients attribute disgust and fear to neutral faces.

Another investigation (Green et al., 2003) discovered in schizophrenia an early increased sensitivity to and later avoidance of threat related facial expressions like anger and fear. Schizophrenic patients perceived angry emotions more rapidly than controls. They are also changing more rapidly from the happy emotional pole to the angry pole regardless of the 
social context and are not able to make relevant social judgments when the context of conversation changes slightly. It means that they are less sensitive to the social nuances of conversation. According to Huang et al (2008) schizophrenic patients were more alert to angry facial expressions in the process of social expressions being morphed from happy to angry.

According to many other investigations, schizophrenic patients have problems in the identification of all facial emotional expressions, either positive or negative (Yamada et al., 2009). In general, the fine differences in face expression recognition are less prominent in schizophrenic patients (Seiferth et al., 2009). In comparison to healthy subjects, patients poorly recognize more complex facial expressions of emotions (Alfimova et al., 2009). Patients performed worse that control subjects on recognition of all emotions and neutral faces combined, including mild and extreme emotional expressions (Kohler et al., 2003). Patients showed less benefit from increased intensity for all emotions combined, especially for fear. Happy faces were often recognized as neutral and even as sad, while sad faces were misrecognized as neutral or disgusted. Angry faces were most commonly misrecognized as neutral, or disgusted, or fearful. Fearful faces were recognized as neutral, or disgusted, or sad. At the same time, patients misidentified neutral cues as emotionally valued with a negative bias (Kohler et al., 2003). Thus, patients seem to be confused with any facial emotional expressions. According to Tsoi et al. (2008) and Pomarol-Clotet et al. (2009) the reaction time on all emotional expressions is increased in patients.

Turetsky et al. (2007) have found that controls performed better than schizophrenic patients in recognizing all facial emotions, and authors come to the conclusion that affect recognition deficit for happy and sad expressions discrimination is secondary to faulty structural encoding of faces. Kohler et al. (2000) have shown that it is difference between genders in facial emotional recognition task performance: male patients perform it relatively worse than females.

Finally, according to some investigations (Pomarol-Clotet et al., 2009 ) schizophrenic patients do not show deficit in emotion identification although nevertheless the process of identification requires more time (see also Tsoi et al. 2008) and patients are less sensitive to the intensity of facial emotional expression (Pomarol-Clotet et al., 2009). According to Seiferth et al (2009), a sensitivity to emotions in patients is not lost, however the task to discriminate emotions and to ignore faces that display emotions that is not necessary to recognize is too difficult for patients.

How is it possible to explain all these contradictory results? From my point of view, there may be different explanations. Schizophrenia is a very complicated disorder with many "faces" and different states and combinations of symptoms. In different states the mechanisms of face expressions recognition may be different. In addition, the comparison between the recognition of different emotional expressions inside the group of patients may give different results from the comparison of recognition the same emotional expressions between patients and healthy subjects.

Schizophrenic patients may be in general less sensitive to any emotional expressions either positive or negative than healthy subjects are. It may be an outcome of emptiness of the world of human interrelationships for the patients. If they in most cases perceive a world of human relationships as a big gray spot than the difference in perception of different emotional expressions of other people is de-evaluated. This explanation corresponds also to the decreased sensitivity to the meaning of facial expressions in social context and to the decreased sensitivity to the artificial distortion of faces: as it was mentioned, patients often accepted these distorted faces as normal. It is understandable if patients are unable to create empathic relationships with other people and if they perceive human faces as inanimate objects.

However, such decreased sensitivity to emotional feelings of other people may be in patients on different level, and on the other hand it does not exclude the sensitivity (and even high sensitivity) not as a sign of empathy but as a sign of the patients' own vulnerability. It is 
a difference between feeling of a pain or of happiness of other subject and a feeling of own pain.

When patients display a relatively increased sensitivity to positive vs. negative expression, it can be explained by two different mechanisms. It may be an activity of the defense mechanism that protects subject from the negative experience he cannot integrate (see later) - "don't touch me". Or it may be the orientation on the very definite simple facial features like smile that helps to form a definite concept - what this form of lips means without any feeling of embodied simulation and empathic relationships.

The opposite result - the relatively higher sensitivity to the negative vs. positive expression - can be explained in the same frame of reasons. Many patients prescribe negative expressions like anger, fear and disgust to the objectively neutral faces and at the same time they are unable to recognize real negative expressions. It is possible to suggest that due to their insensitivity to "humanness" in human relationships they found the world of human being (represented by the vivid human face even with the neutral emotional expression) as something strange and potentially dangerous. Although patients perceive faces in the same way as objects, these strange 'objects" are still different from other objects and due to their vivid nature they cause straining. They evoke fear and anxiety because patients do not feel the nature of face expression and accept it as something not understandable (Morris et al., 2009). It is difficult for patients to understand the intentions of other people and it can cause the suspiciousness. This can be a reason of the prescription of the disgust and anger to the neutral faces, or it can be a projection of the patients' inner state. What patients accept as a cause of fear healthy subjects are estimating as neutral expressions (Tremea et al., 2009). (On the other hand, it may be difficult for patients to recognize Joyful expression and expression of happiness because patients have no self-identifications with these feelings and they are able to recognize them only in a conceptual way, as I have already explained).

Thus, if in patients the ability to react normally on the essence of human face is absent, this essence nevertheless produces an emotional tension as something that subject is unable to integrate into his/her inner world.

In order to preserve sensitiveness to the information that causes anxiety and frustration and at the same time not to lose the adaptive behavior, person has to integrate this information with his world image and Self Image. It is the right frontal lobe that is responsible for this integration however exactly this brain structure is functionally inefficient in schizophrenic patients (Rotenberg, 2004, 2007).

The inability to recognize the face and its emotional expression may be an outcome of the inability to integrate separate features of the image into the holistic gestalt (Doninger et al., 2002). In schizophrenic patients men are less sensitive to the facial expressions than women. At the same time, healthy women are reacting more on the local features of the complex visual stimuli than on their global entities (Roalf et al., 2006). It is possible to suggest that men may be more vulnerable to the disturbance of the right hemisphere functions in psychiatric diseases exactly because healthy men are in general more "right-hemispheric" (see Rotenberg, 2004).

According to Johnson et al. (2005), the disturbance of the global perceptual grasping is the common feature of schizophrenic patients and does not depend on the difference between genders. However, because women are in general more predisposed to the left hemispheric style of perception of local features, including faces, they may be less vulnerable to the dysfunction of the right hemisphere caused by the disease. Probably the problem of schizophrenic patients is not a specific disability to recognize the expression of the face but the deficiency of global perception? Kucharska-Pietura et al. (2009) beliefs that the distorted recognition of facial emotional expression may be a consequence of the deficient face recognition and for this reason patients are also less able to recognize famous subjects. From my point of view this approach ignores a very specific essence of human interrelationships that is a core of essential face and face expression recognition.

The feeling of danger while observing the neutral face expression is still not so strong to activate the perceptual defense or repression. But it is strong enough to prescribe to this face 
negative emotions (like a form of delusions). More definite negative expressions can already activate psychological defense. This approach can explain a seeming paradox when schizophrenic patients prescribe to the neutral faces negative expressions while are unable to recognize obvious negative expressions.

The less patients are able to accept the world, and especially the world of human relationships, in its polysemantic nature, and to integrate their own feelings and experiences, the less protected they are.

\section{CONCLUSION}

The present essay contains the following propositions:

1. The identification of human faces, and especially previously unfamiliar faces, is not based (with the exception of some extraordinary exotic cases) neither on the estimation of the particular single features of the face nor on their configurations that can be calculated and displayed as a relatively simple frame. Human faces differ from any objects and in opposite to objects the inversion of faces disturbs their identification.

2. The recognition of human face depends on the ability of the observer to create holistic and polydimensional virtual relationships with the owner of this face ( the observed person) and these relationships include the previous experience of the observer in the domain of the interpersonal communications. It may explain the implicit integrative conclusion often made by observer according to the face of the unfamiliar subject with a neutral face expression - whether the owner of this face is pleasant or unpleasant and even dangerous. This ability to create immediately complicated polysemantic relationships is a function of the right hemisphere.

3. The similar mechanism is responsible for the recognition of different facial emotional expressions, especially of complicated and vague expressions that need emotional contact (at least virtual and one-side directed) of observer with the observed person, in order to be understood correctly. For instance, in order to feel that the smile is false or represents not a feeling of joy but a feeling of superiority it is necessary to get the holistic impression about the observed subject. However, in more simple cases of the conventional and standard facial expressions (that are often used in the process of investigations) facial expressions may be recognized on the basis of local features of the face without interpersonal contact (when smiling lips are estimated as a sign of joy and frown brow as a sign of anger). It is a conceptualization of the facial expression, and when the real emotional state beyond such expressions is more complicated this conceptualization may cause mistakes in interpretation. It is worth to stress that such conceptualization can be used in the process of estimation of the relatively simple facial expressions but not in the process of recognition of the unfamiliar faces.

4. Another reason of the difficulties and mistakes in recognition of emotional expressions (negative) may be a psychological defense of highly sensitive persons or of persons who are in close relationships with the observed subject.

5. The recognition of famous faces may be based on the conceptualized socially determined associations, that are monosemantic and different from the mechanism of polydimensional interpersonal relationships that are used in the process of the perception of the unfamiliar faces that have to be further recognized.

6. The process of the recognition of subject's own face is based predominantly on the right-hemispheric Self Image and partly on the left hemispheric Self Concept. If the Self-Concept dominates in this process the own face may be mixed with the familiar face (how it happens in patients with suppressed right hemisphere and in schizophrenia). 
7. For schizophrenic patients due to the right hemisphere insufficiency human face is losing its unique human essence and quality and is accepted as an object. Schizophrenic patients do not create the virtual polydimensional relationships with the observed subject in order to integrate this subject in their own polysemantic world like it happens in healthy subjects. However, human face is for the patient a strange object that is alien and not understandable, and just because of such perception of the face human faces even with neutral expression can cause emotional tension in schizophrenia. Probably due to this mechanism schizophrenic patients are ready to prescribe to the neutral observed faces negative expressions like anger, fear or disgust. At the same time, paradoxically, the same negative emotions being really expressed are often not recognized by the patients. My suggestion is that when they are really expressed they are crossing the "red line" for acceptance. And positive emotions are often not recognized by these patients due to the lack of identification with positive feelings.

\section{REFERENCES}

Adolphs, R. (2002). Recognizing emotion from face expressions: psychological and neurological mechanisms. Behavioral and Cognitive Neuroscience Reviews, 1, 21-62.

Alfimova, M.V., Abramova, L.I., Barhatova, A.I., Yumatova, P.E., Lyanchenko, G.L., Golimbet, V.E. (2009). Facial affect recognition deficit as a marker of genetic vulnerability to schizophrenia. The Spanish Journal of Psychology, 12, 46-55.

An, S.K., Lee, S.J., Lee, C.H., Cho, H.S., Lee, P.G., Lee, C.I., Lee, E., Roh, K.S., Namkoong, K. (2003). Reduced P3 amplitudes by negative facial emotional photographs in schizophrenia. Schizophrenia Research, 64, 2-3, 125-135.

Bar, M \& Neta, M. (2006a). Humans prefer curved visual objects. Psychological science, 17, 645-648.

Bar, M. , Neta, M., \& Linz, H. (2006b). Very first impressions. Emotion, 6, 269-278.

Blau, V.C., Maurer, U., Tottenham, N., McCandliss, B.D. (2007). The face- specific N170 component is modulated by emotional facial expression. Behavioral and Brain Functions, 23(3), 7.

Bleich-Cohen, M., Strous, R.D., Even, R., Rotshtein, P., Yovel, G., Iancu, I., Olmer, A., Hendler, T. (2009). Diminished neural sensitivity in irregular facial expression in first-episode schizophrenia. Human Brain Mapping, 30, 2606-2616.

Caharel, S., Courtay, N., Bernard, C., Lalonde, R., Rebai M. (2005). Familiarity and emotional expression influence an early stage of face processing: an electrophysiological study. Brain and Cognition, 59, 96-100.

Calder, A.J., \& Jansen, J. (2005). Configural coding of facial expressions: The impact of inversion and photographic negative. Visual Cognition, 12, 495-518.

Calder, A.J., \& Young, A.W. (2005). Understanding the recognition of facial identity and facial expression. Nature Reviews Neuroscience, 6, 641-651.

Calder, A.J., Keane, J., Young, A.W., Dean M. (2000). Configural information in facial expression perception. Journal of Experimental Psychology: Human perception and performance, 26, 527-551.

Craik, I.M., Moroz, T., Moscovitch, M., Stuss D.T., Wincour, G., Tulving, E., Kapur, S. (1999). In search of the self: A positron emission tomography study. Psychological Science, 10, 26-34

Daprati, E., Franck, N., Georgieff, N., Proust, J., Pacherie, E., Dalery, J., Jeannerod, M. (1997). Looking for the agent: an investigation into consciousness of actijn and self-consciousness in schizophrenic patients. Cognition, 65, 1, 71-86.

Doniger, G.M., Silipo, G., Rabinowicz, E.F., Snodgrass, J.G., Javitt, D.C. (2001). Impaired sensory processing as a basis for object-recognition deficits in schizophrenia. American Journal of Psychiatry, 158, 1818-1826.

Doniger, G.M., Foxe, J.J., Murray, M.M., Higgins, B.A., Javitt, D.C. (2002). Impaired visual object recognition and dorsal/ventral stream interaction in schizophrenia. Archive General Psychiatry, 59, 1011-1020.

Gallup, J.G.G. (1982). Self-awareness and the emergence of mind in primates. Americal Journal of Primatology, 2, 237-248.

Ghuman A.S., Bar M. (2006). The influence of non-remembered affective associations on preference. Emotion, 6, 215-223. 
Green, M.J., Williams, L.M., Davidson, D. (2003). Visual scanpaths to threat-related faces in deluded schizophrenia. Psychiatry Research, 119, 271-285.

Happe, F., Brownell, H., Winner, E. (1999). Acquired “Theory of mind" impairments following stroke. Cognition, 70, 211-240.

Harrington, A., Oepen, G., Spitzer, M. (1989). Disordered recognition and perception of human faces in acute schizophrenia and experimental psychosis. Comprehensive Psychiatry, 30, 376-384.

Haxby, J.V., Hoffman, E.A., Gobbini, M.I. (2000). The distributed human neural system for face perception. Trends in Cognitive Sciences, 4, 223-233.

Huang, J., Chan, R.C.K., Lu, X., Tong, Z. (2009). Emotion categorization perception in schizophrenia in conversations with different social contexts. Australian and New Zealand Journal of Psychiatry, 43, 438-445.

Irani, F., Platek, S.M., Panyavin, I.S., Calkins, M.E., Kohler, Ch., Siegel, S.J., Schachter, M., Gur, R.E., Gur, R.C. (2006). Self-face recognition and theory of mind in patients with schizophrenia and firstdegree relatives. Schizophrenia Research, 88, 151-160.

Johnson, S.C., Lowery, N., Kohler, C., Turetsky, B.I. (2005). Global-local visual processing in schizophrenia: evidence for an early visual processing deficit. Biological Psychiatry, 58, 937-946.

Joshua N., \& Rossell S. (2009). Configural face processing in schizophrenia. Schizophrenia Research, 112, 99-103.

Keenan J.P., McCutcheon B., Freund S., Gallup Jr., Sanders G., Pascual-Leone A. (1999). Left hand advantage in a self-face recognition task. Neuropsychologia, 37, 1421-1425.

Keenan J.P., Nelson A., Pascual-Leone A. (2001). Self-recognition and the right hemisphere. Nature, 409, 305.

Kohler, C.G., Bilker, W., Hagendoorn, M., Gur, R.E., Gur, R.C. (2000). Emotion recognition deficit in schizophrenia: association with symptomatology and cognition. Biological Psychiatry, 48, 127-136.

Kohler, Ch. G., Turner, T.H., Bilker, W.B., Brensinger, C.M., Siegel, S.J., Kanes, S.J., Gur, R.E., Gur, R.C. (2003). Facial emotion recognition in schizophrenia: intensity effect and error pattern. American Journal Psychiatry, 160, 1768-1774.

Kostandov E.A., Genkina O.A. (1976). Hemisphere asymmetry of evoked electrical activity of the cerebral cortex to letter and nonverbal stimuli. Zhurnal Vysshey Nervnoy Deyatelnosti, 26, 21-29.

Kucharska-Pietura, K., David, A., Dropko, P., Klimowski, M. (2002). The perception of emotional chimeric faces in schizophrenia: Further evidence of right hemisphere dysfunction. Neuropsychiatry, Neuropsychology, Behavioral Neurology, 15, 72-78.

Leppanen, J.M., Niehaus, J.H., Koen, L., Schoeman, R., Emsley, R. (2008). Allocation of attention to the eye and mouth region of faces in schizophrenia patients. Cognitive Neuropsychiatry, 13, 505-519.

Morris, R.W., Weickert, C.Sh., Loughland, C.M. (2009). Emotion face processing in schizophrenia. Current Opinion in Psychiatry, 22, 140-146.

Niedenthal, P.M., Mermillod, M., Maringer, M., Hess, U. (2010). The Simulation of Smiles (SIMS) Model: Embodied Simulation and the Meaning of Facial Expression. Behavioral and Brain Sciences, 33, 445-446.

Norton D., McBain R., Holt D.J., Ongur D., Chen Y. (2009). Association of impaired facial affect recognition with basic facial and visual processing deficits in schizophrenia. Biological Psychiatry, 65, 1094-1098.

Obayashi, Ch., Nakashima, T., Onitsuka, T., Mackawa, T., Hirano, Y., Hirano, Sh., Oribe, N., Kaneko, K., Kanba, Sh, Tobimatsu, Sc. (2009). Decreased spatial frequency sensitivities for processing faces in male patients with chronic schizophrenia. Clinical Neuropsychology, 120, 1525-1533.

Orbach, J., Traub, A.C., Olson, R. (1960). Psychological studies of body-image: II. Normative data on the adjustable body-distorting mirror. Archives of General Psychiatry, 14, 41-47.

Platek, S.M., Gallup, G.G. (2002). Self-facerecognition is affected by schizotypal personality traits. Schizophrenia Research, 57, 311-315.

Platek S.M., Myers T.E., Critton S.R., Gallup G.G. (2003). A left-hand advantage for self-description: the impact of schizotypal personality traits. Schizophrenia Research, 65, 147-151.

Platek, S.M., Keenan, J.P., Gallup, G.G., Mohamed, F.B. (2004). Where am I? The neurological correlates of self and other. Cognitive Brain Research, 19, 114-122.

Platek, S.M., Wathne, K., Tierney, N.G., Thomson, J.W. (2008). Neural correlates of self-face recognition: An effect-location meta-analysis. Brain Research, 1232, 173-184.

Pomarol-Clotet, E., Hynes, F., Ashwin, C., Bullmore, E.T., McKenna, P.J., Laws, K.R. (2010). Facial emotion processing in schizophrenia: a non-specific neuropsychological deficit? Psychological Medicine, 40, 911-919.

Preilowski, B. (1977). Self-recognition as a test of consciousness in left and right hemisphere of "splitbrain" patients. Activitas Nervosa Superior (Praha), 19 (suppl. 2) 343-344. 
Roalf, D.R., Lowery, N., Turetsky, B.I. (2006). Behavioral and physiological findings of gender differences in global-local visual processing. Brain and Cognition, 60, 32-42.

Rotenberg, V.S. (1979). Word and image: The problem of context. Dynamische Psychiatrie/Dynamic Psychiatry, 59, 494-498.

Rotenberg, V.S. (1982). Funktionelle Dichotomie der Girnhemispharen und die Bedeutung der Suchaktivitat fur physiologische und psychologische Processe. In. G. Ammon (Ed.) Handbuch der Dynamischen Psychiatrie, Bd.2, 276-336.

Rotenberg, V.S. (1985). Sleep dreams: Cerebral dominance and creation. Pavlovian Journal of Biological Science, 20, 53-58.

Rotenberg, V.S. (1994). An integrative psychophysiological approach to brain hemisphere functions in schizophrenia. Neuroscience and Biobehavioral Reviews, 18, 487-495.

Rotenberg, V.S. (2004). The peculiarity of the right hemisphere function in depression: Solving the paradoxes. Progress in Neuro-Psychopharmacology and Biological Psychiatry, 28, 1-13.

Rotenberg, V.S. (2007). The asymmetry of the frontal lobe functions and the fundamental problems of mental health and psychotherapy. Dynamische Psychiatrie/Dynamic Psychiatry, 40, 51-68.

Rotenberg, V.S. (2008). Functional brain asymmetry as a determinative factor in the treatment of depression: Theoretical implications. Progress in Neuro-psychopharmacology and Biological Psychiatry, 32, 1772-1777.

Saugstad, L.F. (1998). Cerebral lateralization and rate of maturation. International Journal of Psychophysiology, 28, 37-62.

Schwartz, B.L., Marvel, Ch.L., Drapalski, A., Rosse, R.B., Deutsch, S. I. (2002). Configural processing in face recognition in schizophrenia. Cognitive Neuropsychiatry, 7, 15-39.

Seiferth, N.Y., Panly, K., Kellerman, Th., Jonshah, N., Off, G., Herpetz-Dahlmann, B., Kircher, T., Schneider, F., Habil, U. (2009). Neuronal correlates of facial emotion discrimination in early onset schizophrenia. Neuropsychopharmacology, 34, 477-487.

Shin, Y.-W., Na, M.H., Ha T.H., Kang D.-H., Yoo S.-Y., Kwon J.S. (2008). Dysfunction inconfigural face processing in patients with schizophrenia. Schizophrenia Bulletin, 34, 538-543.

Silver, H., \& Bilker, W. (2009). Goodman C. Impaired recognition of happy, sad and neutral expressions in schizophrenia is emotion, but not valence, specific and content dependent. Psychiatry Research, 169, 101-106.

Simons, D.J., \& Levin D.T. (1998). Failure to detect changes to people during a real world interaction. Psychosomatic Bulletin and Review, 5, 644-649.

Snyder, S., Rosenthal, D., Taylor, I.A. (1961). Perceptual closure in schizophrenia. Journal of Abnormal and Social Psychology, 63, 131-136.

Stuss, D.T., Gallup, G.G., Alexander, M.P. (2001). The frontal lobes are necessary for theory of mind. Brain, 124, 279-286.

Sugiura, M., Watanabe, J., Maeda, Y., Matsue, Y., Fukuda, H., Kawashima, R. (2005). Cortical mechanisms of visual self-recognition. Neuroimage, 24, 143-149.

Tremeau, F. (2006). A review of emotion deficits in schizophrenia. Dialogues in Clinical Neuroscience, $8,59-70$.

Tremea, F., Antonius, D., Goggin, M., Czobok, P., Butler, P., Malaspina, D., Gorman, J.M. (2009). Emotion antecedents in schizophrenia. Psychiatry Research, 169, 43-50.

Tsoi, D.T., Kwang-Hyuk, L., Khokhar, W.A., Mir, N.U., Swalli, J.S., Gee, K.A., Pluck, G., Woodruf, P.W.R. (2008). Is facial emotion recognition impairment in schizophrenia identical for different emotions? A signal detection analysis. Schizophrenia Research, 99, 263-269.

Tsoi, D.T., Lee, K.-H., Khokhar, W.A., Mir, N.U., Swalli, J.S., Gee, K.A., Pluck, G., Woodruff, P.W.R. (2008). Is facial emotion recognition impairment in schizophrenia identical for different emotions? A signal detection analysis. Schizophrenia Research, 99, 263-289.

Turetsky, B.I., Kohler, Ch.G., Indersmitten, T., Bhati, M.T., Charbonnier, D., Gur, R.C. (2007). Facial emotion recognition in schizophrenia: When and why does it go awry? Schizophrenia research, 94, 253-263.

Turk, D.J. , Heartherton, T.F., Kelley, W.M., Funnel, M.G., Gazzaniga, M.S., Macrae, C.N. 2002, Mike or me? Self-recognition in a split-brain patient. Nature Neuroscience, 5, 841-842.

Walker, E., Marwit, S.J., Emery, E. (1980). A cross-sectional study of emotion recognition in schizophrenics. Journal of Abnormal Psychology, 89, 428-436.

Walther, S., Federspiel, A., Horn, H., Bianchi, P., Wiest, R., Wirth, M., Strik, W., Muller, Th.J. (2009). Encoding deficit during face processing within the right fusiform face area in schizophrenia. Psychiatry Research: Neuroimaging, 172, 184-191. 
Yamada M., Ueda K., Namiki Ch., Hirao K., Hayashi T., Onigashi Y., Mirai T. (2009). Social cognition in schizophrenia: similarities and differences of emotional perception from patients with facial frontal lesions. European Archives of Psychiatry and Clinical Neurosciences, 259, 227-233.

Yin, R.K. (1969). Looking at upside-down faces. Journal of Experimental Psychology, 81(1), 141.

Young, A.W., Hellawell, D., Hay, D.C. (1987). Configurational information in face perception. Perception, 16, 747-759. 